
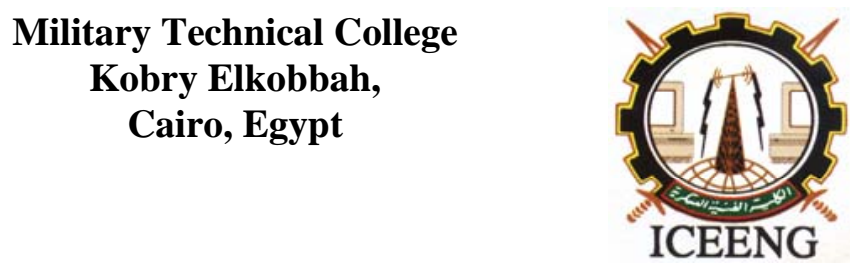
$5^{\text {th }}$ International Conference on Electrical Engineering ICEENG 2006

\title{
MODELING AND TESTING OF COMPUTER DISTANCE RELAY USING COMPUTER SIMULATION
}

\author{
Abdel Rahman A. Khatib ${ }^{\star}$
}

\begin{abstract}
The paper presents modeling and testing of computer distance relay using MATLAB/PSCAD. The relay has been tested in $345 \mathrm{kV}$ parallel overhead transmission lines that include the mutual coupling. The paper is demonstrating the relay's capability to detect the fault at different zones, producing a tripping output for different fault conditions. The concepts of traditional distance relaying were used and three zones were defined for the transmission system to be protected by the relay. Different fault types were simulated occurring at distinct locations within the transmission system. The computer relay is developed using MATLAB and the transmission line model is implemented using EMTDC / PSCAD.
\end{abstract}

KEYWORDS - Distance mho Relay, PSCAD, Relay Model, Protection, and MATLAB

\section{INTRODUCTION}

Digital computer relay began in the 1960's [1]. The functions complexity of the digital relays program requires interactive tools [2] to verify and test the algorithms of these functions.

Transient approaches in relaying and testing are not new. However, their broad approval was postponed for several reasons as the use of network system simulators, and the expensive cost of these simulators. This has changed with the arrival of the first digital simulators in the late eighties and early nineties [3]. One of the most commonly used non real-time simulator as Electromagnetic transients program [3] is PSCAD [4]

These Electromagnetic transients make the testing and simulating of digital protection [5] much more easy and efficient. Many protection schemes realization [6-7] are introduced. Besides the realization of protection schemes, the Electromagnetic simulator will help in producing Educational tools [8] to help the student to understand the protection behavior details of digital relays.

With the help of one of the fastest Engineering tool program like the MATLAB [9], Modeling and testing of digital protection becomes more efficient and easier to implement [10].

In this paper well-established simulator for distance relay with MATLAB/PSCAD is implemented. Quite different options are offered for better understanding the algorithm detail [11] of a digital distance protection.

\footnotetext{
*Assistant Professor at Shorouk Academy, Cairo, Egypt.
} 
The concepts of traditional distance relaying [12] were used and three zones were defined for the transmission system to be protected by the relay. Different fault characteristics (phase to phase, phase to ground, three-phase, etc.) were simulated occurring at distinct locations within the transmission system. The relay should be able to produce a correct decision to faults occurring in zone 1 , zone 2 , zone 3 , and out of its protection zone.

EMTDC/ PSCAD software is used to get the pre-fault, fault and post-fault condition data and once this was available, the signal conditioning process started. MATLAB software is employed to implement different relay modules using modules oriented programming. Several MATLAB programs were written, having a "main" program controlling the function of other subroutines.

The relay algorithm has been tested in a $345 \mathrm{kV}$ three phase transmission line and the expected results have been obtained.

\section{TEST SYSTEM CONFIGURATION}

A $345-\mathrm{kV}$ system in which the relay was tested is shown in Fig. 1. It is constituted by 3 transmission lines, a generator and a motor. Two of the transmission lines are parallel lines (100 miles) that carry flows from generator A to motor B and the third line (50 miles) is unloaded line that has be used for testing the second zone of the relay

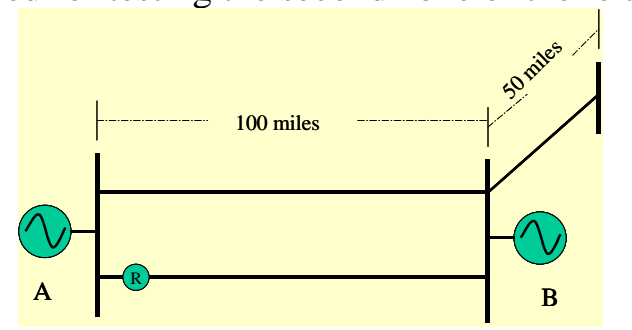

Fig. 1: one-line schematic for system under study

The relay " $R$ " is in fact a relay system, since it is composed of six relays, i.e., a-b relay, b-c relay, c-a relay; for the phase relays, and a-g , b-g, c-g for the ground relays. Even though in the present times the modern digital relays integrate the six relays in one package we decided to analyze the specific response of each relay to the different fault characteristics and locations. By doing this, we acquired a satisfactory insight of the phenomena and in most of the cases we could classify the type of fault.

The system is composed of 10 "pi" segments for the parallel lines and five "pi" sections for the other line. The model takes into consideration mutual coupling among the phases of the lines. The mutual coupling modeling required some work due to the EMTDC configuration since a special matrix had to be constructed. Care was taken by checking the amount of power being transmitted by the parallel lines. Basically we adapted the power to a real practical value in order to avoid difficulties for the zones settings, in particular Zone three, since the load current may encroach into this remote back-up protection zone, resulting in a relay operation for load conditions. The line parameters we used are as follows:

$$
\begin{aligned}
& Z_{\text {self }}=2.73+j 10 \Omega / 10 \text { miles } \\
& Z_{\text {mutual }}=1.7136+j 4.07 \Omega / 10 \text { miles } \\
& Z_{1}=Z_{\text {self }}-Z_{\text {mutual }}=10.197+j 59.3 \Omega / 100 \text { miles } \\
& C=0.1298 \mu \mathrm{F} / 10 \text { miles }
\end{aligned}
$$




\section{RELAY MODULES}

The stages or modules in which the relay was divided are shown in Fig. 2. In this section description and objective of each stage is explained.

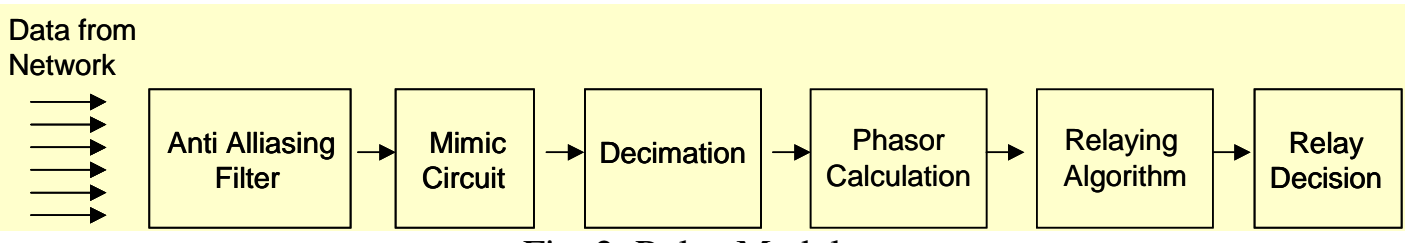

Fig. 2: Relay Modules

\section{Data from Network}

The first stage was to obtain the pre-fault, fault and post-fault condition data from the network, i.e., three currents and three voltages required as an input to the relay. EMTDC/ PSCAD is used in order to construct an accurate model to the transmission line.

Different faults type and location were tested in the system, which provide the flexibility of changing the fault characteristics, such as fault type, time of occurrence, duration time, etc. Minimum fault resistance allowed by the EMTDC has been used. Each case was saved in a data file. This was the final goal of the first module. The data files are read by a MATLABbased interface.

\section{Anti-Alliasing Filter}

Anti-Alliasing Filter is the second stage according to Fig. 2, the order does not affect the simulation results since we are working with linear models in a simulation environment. The objective of this module is to cut down the high frequency components that may be present in the signal. The Anti-Alliasing filter used was a low pass, two stages RC filter.

\section{$\underline{\text { Mimic Circuit }}$}

The mimic circuit is the third stage according to Fig. 2. In real life, the mimic circuit is actually a circuit that is connected in the secondary of the current transformers. It is used in order to eliminate the decaying exponential component, commonly referred as "decaying dc offset" from the current signal. In our simulation environment we eliminate the "dc offset" by using an RL mimic circuit with an Rmimic/Lmimic ratio value equal to the transmission line R/L value [1].

\section{Decimation}

Decimation is normally called to the process of reducing the sampling frequency. In the real life A/D converters are used to convert the analogue signals to digital, however, in this simulation only digital signals are available. The data coming form EMTDC/PSCAD, which was passed through the Anti-Alliasing and mimic filters, is digital data sampled at a very high sampling rate, i.e., 600 samples per cycle. Since the sampling rate chosen to calculate the phasors was 12 samples per cycle, or $720 \mathrm{~Hz}$, the decimation process was required. Simple resampling technique is chosen by picking one signal each 50 signal. 


\section{Phasor Calculation}

Once the sampled data was at the correct sampling rate the next stage was to calculate the phasors. The recursive formula was used for 1 cycle relay. This technique requires the first 12 samples per cycle in order to calculate the phasor. Once a new sample arrives, the oldest sample is dropped and the new one is added, allowing having a fixed phasor in the plane. Phasor calculations are meaningless for some conditions such as for the first samples (1-11 for 12 samples per cycle sampling rate), or when the data window contains samples pre fault condition mixed with faulted conditions as will be seen in the result section.

For the fast relay one cycle algorithm is considered long time, so the fractional relay is preferred. A general matlab program using non-recursive algorithm can be used to calculate the phasor for any sampling rate and any speed is also implemented

\section{$\underline{\text { Relay Algorithm }}$}

Once the three voltage and three current phasors are calculated, the next step is to compute the impedance as seen in Fig. 3. According to the distance principle, the relay will react to the apparent impedance as seen from its terminals.

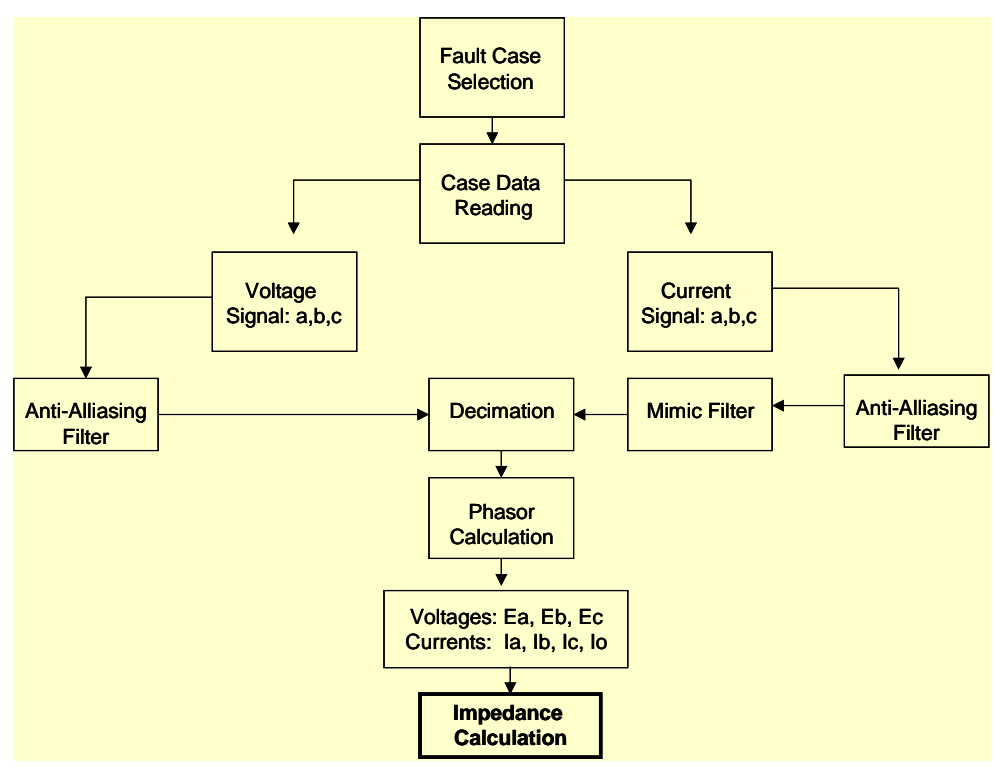

Fig. 3: Main Relay Program Algorithm

The impedance was computed using the following equations.

For the phase-phase relays:

Relay a-b: $\frac{E_{a}-E_{b}}{I_{a}-I_{b}}, \quad$ Relay b-c: $\frac{E_{b}-E_{c}}{I_{b}-I_{c}}$, Relay c-a: $\frac{E_{c}-E_{a}}{I_{c}-I_{a}}$

For the phase-ground relays:

Relay a-g: $\frac{E_{a}}{I_{a}+m \cdot I_{0}}$, Relay b-g: $\frac{E_{b}}{I_{b}+m \cdot I_{0}}$, Relay c-g: $\frac{E_{c}}{I_{c}+m \cdot I_{0}}$ 
Where

$$
\begin{array}{ll}
E_{a}, E_{b}, E_{c} & \text { are the phase voltages phasors } \\
I_{a}, I_{b}, I_{c} & \text { are the phase currents phasors } \\
I_{0} & \text { is zero sequence current phasors } \\
m=\frac{3 z_{\text {mutual }}}{Z_{\text {self }}-Z_{\text {mutual }}} & \text { is a correction factor }
\end{array}
$$

\section{$\underline{\text { Relay Decision }}$}

Clearly the goal in this module is to be sure that the each relay was reacting correctly to each fault. The distance relay characteristic chosen was a mho relay, taking advantage of its directional capability. The first step of this stage is to set the relay setting zones. The settings are expressed as a function of the transmission line impedance, ZL which magnitude is 60.17 ohms as shown in table 1

Table 1: Digital Relay Settings

\begin{tabular}{|c|c|}
\hline Zone & Impedance setting as a function of ZL \\
\hline 1 & $\mathrm{Z} 1=.80 * \mathrm{ZL}$ \\
\hline 2 & $\mathrm{Z} 2=200^{* *}$ \\
\hline 3 & $\mathrm{Z3}=297^{* *}$ \\
\hline
\end{tabular}

Note: $* *$ These values were calculated after doing a short circuit analysis

The second step of this stage is checking if the impedance seen by the relay within the protection zone or not. The solution to this problem was to restrict the relay decision until FOUR consecutive phasors do not differ from each other by a certain small value. Then the relay decision was restricted by the algorithm shown in Fig. 4.

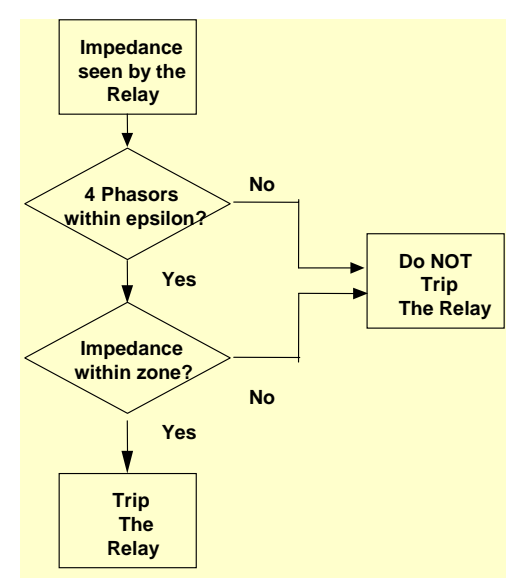

Fig. 4: Relay decision Restriction

\section{RESULTS PRESENTATION USING GRAPHICAL USER INTERFACE (GUI)}

The section was related to the graphical user interface, an appropriate way to present the results. All programs and figures that are called by the GUI are pre-run, so the response is fast. A general way what the graphical user interface capabilities are shows Fig. 5 the main screen of the Graphical User Interface. 


\section{SIMULATION RESULTS}

This section shows with Figures the outputs of each module and verifies the proposed procedures are correct. From all the cases simulated a phase-ground fault at zone one is selected as a sample to show the results.

\section{Single line to ground fault in zone-1}

The voltage signal for phase "a" as an example is shown in Fig. 6. It is evident the voltage decrement after the fault (0.15s) in the lower graph, which is the output of the Anti-Allising filter.

The current signal for phase "a" as an example is shown in Fig. 7.The first plot is the system current signal, the second is the signal after the Anti-Alliasing filter and the third one corresponds to the output of the mimic circuit. The absence of the decaying exponential component, or "dc offset" is evident in the graphs, validating the mimic circuit effect.

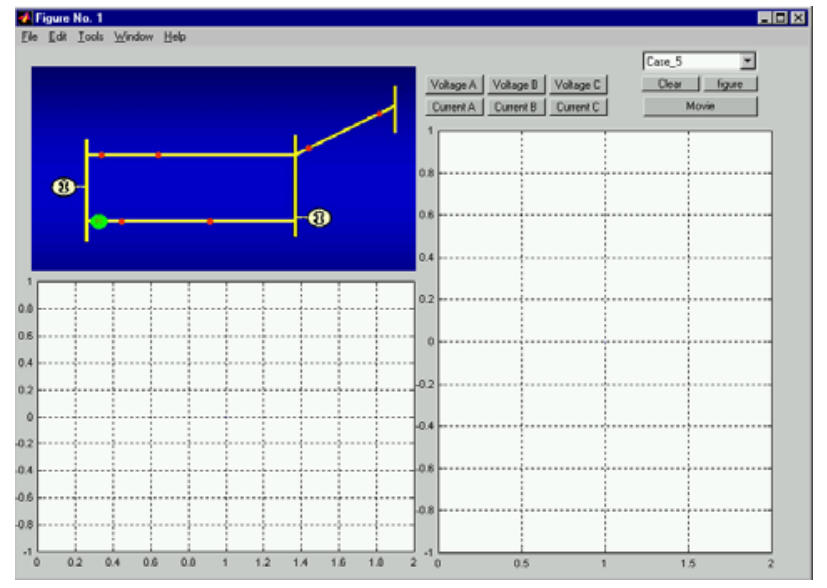

Fig. 5: GUI Main Screen
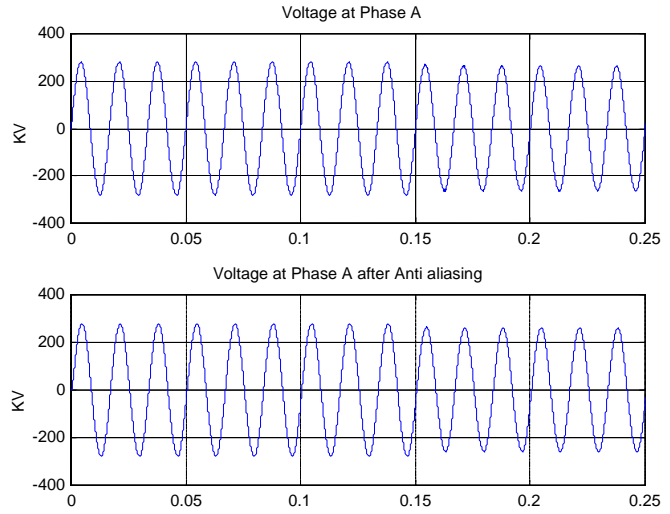

Fig. 6: Voltage at phase 'a' for single line to ground fault at zone-1

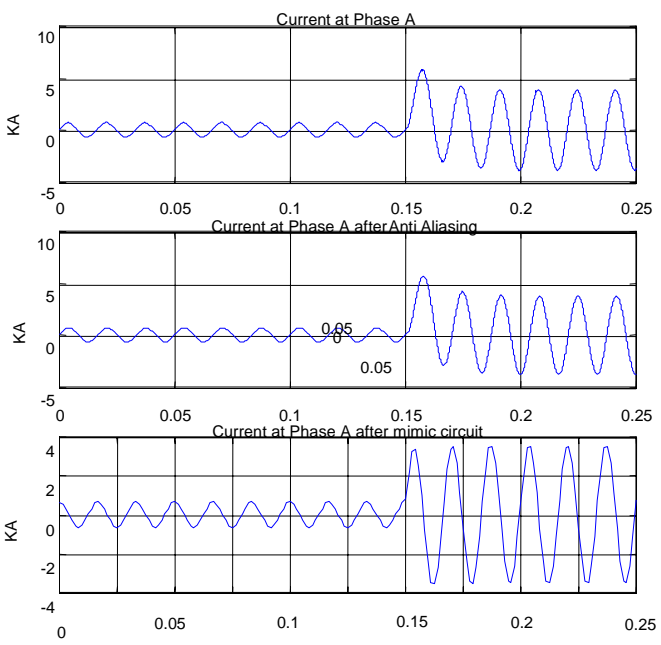

Fig. 7: Current at phase 'a' for single line to ground fault at zone-1 
The magnitude of the impedance seen by the phase and ground relays is shown in Fig. 8 . The upper plot shows the phase relays, i.e., a-b, b-c and c-a, where as the lower one includes the magnitude of the impedance seen by the ground relays, a-g, b-g, c-g. The comments related to the meaningless of phasors, described before are evident by observing these plots, since at the very beginning the phasors differ drastically from each other.
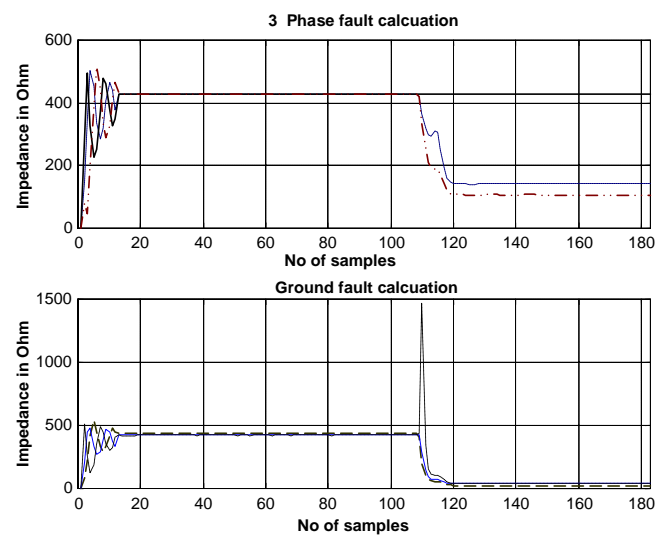

Fig. 8 : magnitude of the impedance for single line to ground fault at zone-1

Fig. (9) shows the response of each relay to a single line to ground fault. The "pulse” shapes response presents the relay reaction, 1 for tripping and 0 for not tripping.

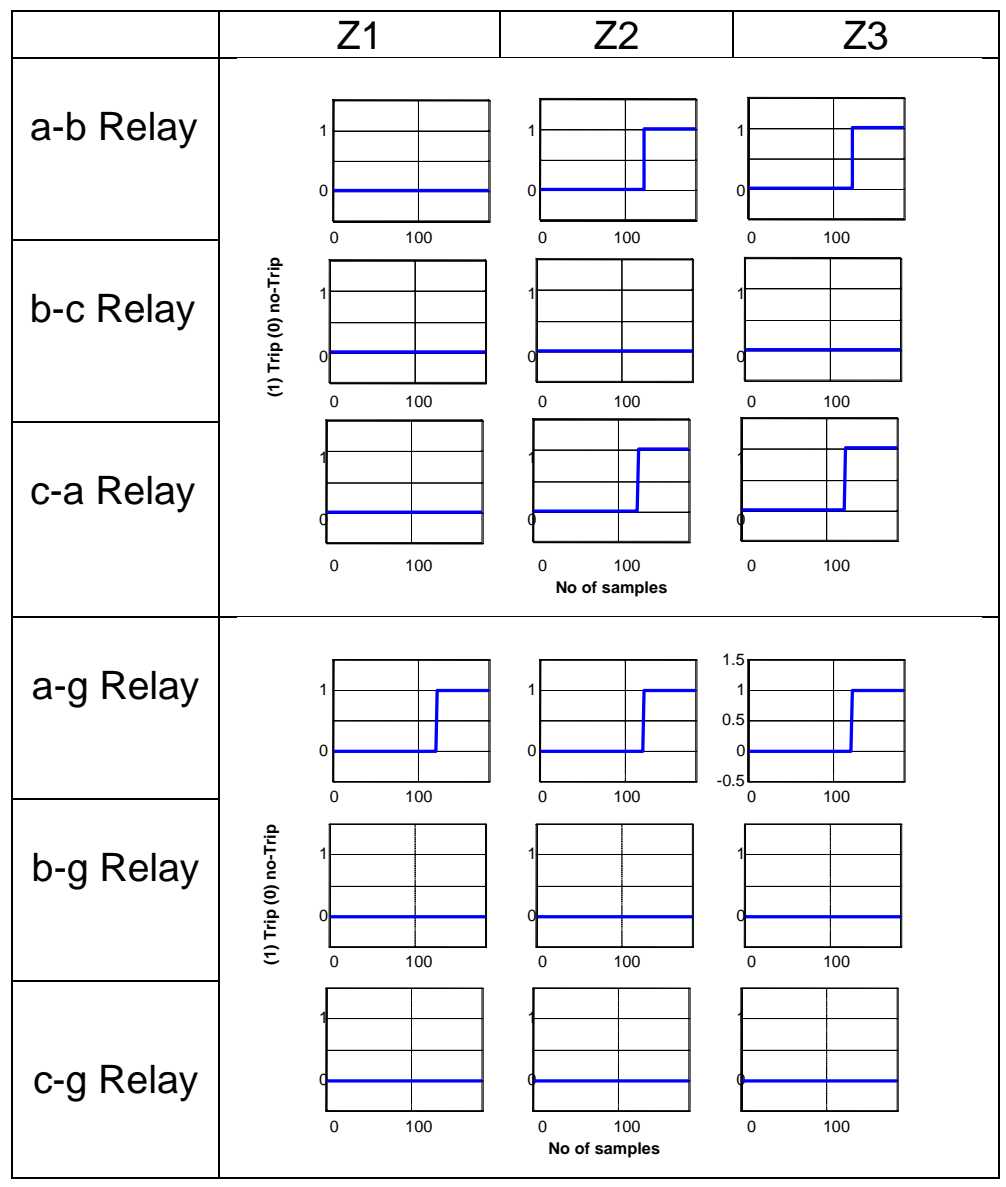

Fig. 9: The response of each relay to a single line to ground fault at zone-1 


\section{CONCLUSIONS}

This paper demonstrates a MATLAB/PSCAD model for a distance relay; the data input for the distance relay is simulated using PSCAD. The relay model and the algorithm of the distance relay has modeled and tested using MATLAB. Although PSCAD/MATLAB model is simulating off-line, it has the advantage that it is able to study details operation distance relay and simulate it in a convenient way. The relay set -that is composed of six relays- has been tested in 345-kV overhead transmission system for different fault type, different time, and locations and relay respond as desired.

\section{REFERENCES}

[1] Arun G.Phadke, James S.Thorp, “Computer Relaying for Power Systems”, John Wiley \& Sons, 1993, pp.112-162.

[2]M. Kezunovic, Q. Chen, "A Novel Approach for Interaction Protection System Simulation", IEEE Trans. on Power Delivery, vol. 12, no. 2, Apr. 1997, pp. 668-674

[3]Alternative Transients Program (ATP) http://www.emtp.org/

[4]Power Systems Computer Aided Design (PSCAD ${ }^{\mathrm{TM}}$ ) website http://pscad.com/

[5]Arvind K. S. Chaudhary, Arun G. Phadke, "Protection System Representation in the Electromagnetic Transients Program", IEEE Trans. on Power Delivery, vol. 9, no. 2 April 1994, pp.700 - 711

[6]J.Y. Heo1, C.H. Kim1, K.H. So1, N.O. Park2 "Realization of Distance Relay Algorithm using EMTP MODELS", International Conference on Power Systems Transients - IPST 2003 in New Orleans, USA

[7]Lee, J.B.Ha, C.W. Jung, C.H. "Development of digital distance relaying algorithm in combined transmission lines with underground power cables ", Power Engineering Society Summer Meeting, vol.1, 15-19 July 2001, pp 611-616

[8]C.H. Kim, M.H. Lee, R.K. Aggarwal, A.T. Johns, "Educational Use of EMTP MODELS for the Study of a Distance Relaying Algorithm for Protecting Transmission Lines", IEEE Trans. on Power System, vol. 15, no. 1, Feb. 2000, pp. 9-15.

[9]The MathWorks, Inc., Getting Started with MATLAB, Natick, MA, March 2005.

[10] Stan Li-Cheng Wu Chih-Wen Liu Ching-Shan Chen, "Modeling and testing of a digital distance relay MATLAB/SIMULINK”, Power Symposium, 2005. Proceedings of the 37th Annual North American,23-25 Oct. 2005, pp 253 - 259

[11] Khorashadi-Zadeh, H. Daneshi, H. "Evaluation and performance comparisons of digital distance protection algorithms ”. Industry Applications Conference, 2004. 39th IAS Annual Meeting, 3-7 Oct. 2004, Vol. 4 pp 2463 - 2468

[12] Stanley H. Horowitz, Arung G. Phadke "Power System Relaying”, Second Edition, 1996. 